\title{
Explicit formulas for Neumann coefficients in the plane-wave geometry
}

\author{
Yang-Hui He, ${ }^{1}$ John H. Schwarz, ${ }^{2}$ Marcus Spradlin, ${ }^{3}$ and Anastasia Volovich ${ }^{4}$ \\ ${ }^{1}$ The University of Pennsylvania, Philadelphia, Pennsylvania 19104 \\ ${ }^{2}$ California Institute of Technology, Pasadena, California 91125 \\ ${ }^{3}$ Princeton University, Princeton, New Jersey 08544 \\ ${ }^{4}$ Kavli Institute for Theoretical Physics, Santa Barbara, California 93106
}

(Received 15 January 2003; published 24 April 2003)

\begin{abstract}
We obtain explicit formulas for the Neumann coefficients and associated quantities that appear in the three-string vertex for type IIB string theory in a plane-wave background, for any value of the mass parameter $\mu$. The derivation involves constructing the inverse of a certain infinite-dimensional matrix, in terms of which the Neumann coefficients previously had been written only implicitly. We derive asymptotic expansions for large $\mu$ and find unexpectedly simple results, which are valid to all orders in $1 / \mu$. Using BMN duality, these give predictions for certain gauge theory quantities to all orders in the modified 't Hooft coupling $\lambda^{\prime}$. A specific example is presented.
\end{abstract}

DOI: 10.1103/PhysRevD.67.086005

PACS number(s): $11.25 . \mathrm{Sq}$

\section{INTRODUCTION}

This paper continues the study of the light-cone gauge string field theory formulation of type IIB superstring theory in the maximally supersymmetric plane-wave geometry [1-7]. Its purpose is to derive and analyze explicit formulas for the Neumann coefficients that enter in the three-string interaction vertex that describes the process in which strings No. 1 and No. 2 join to form string No. 3. In particular [1], this requires an explicit formula for the inverse of a certain infinite-dimensional matrix called $\Gamma_{+}(\mu, y)$ (and defined below). Here, $\mu$ is the mass parameter that appears in the plane-wave metric. It becomes physically meaningful once we specify the coordinate frame, since only the product $P_{-} \mu$ is invariant under a longitudinal boost. The combination $\alpha^{\prime} P_{-}$for the $r$ th string is conventionally denoted $\alpha_{r}$, with the momentum taken to be negative for the outgoing string so that $\Sigma_{r} \alpha_{r}=0$. We make $\mu$ a meaningful dimensionless parameter by choosing a frame for which $\alpha_{3}=-1$. When strings No. 1 and No. 2 join to form string No. 3, string No. 1 carries momentum fraction $\alpha_{1}=y$ and string No. 2 carries momentum fraction $\alpha_{2}=1-y$, with $0 \leqslant y \leqslant 1$.

Previously [4,5], the inverse of $\Gamma_{+}$was determined in terms of a certain infinite component vector called $Y_{m}(\mu, y)=\left(\Gamma_{+}^{-1} B(y)\right)_{m}$ and a scalar function $k(\mu, y)$ $=B^{\mathrm{T}} \Gamma_{+}^{-1} B$. Here $B_{m}(y)$ is a known infinite component vector. (The formula will be given later.) In this paper we obtain explicit formulas for $Y_{m}$ and $k$, and hence also for $\Gamma_{+}^{-1}$. The first step is to derive a first-order differential equation that determines the dependence of $Y_{m}$ on the mass parameter $\mu$. Since the value of $Y_{m}$ for $\mu=0$ is known from previous analysis of the flat-space problem, that knowledge can be used to fix the "initial condition." The resulting integrated expression for $Y_{m}$ is expressed in terms of $k(\mu, y)$, which still needs to be determined. Knowledge of the leading large $\mu$ asymptotic behavior of $Y_{m}$, for all $m$, is sufficient to completely determine that function. The resulting equation involves a certain integral transform. The equation is solved by the inverse integral transform. The particular integral trans- form that appears does not seem to be contained in the standard mathematical references (such as Bateman), but we have not done an exhaustive search of the literature.

The formulas obtained at this point in the analysis are explicit and complete, but they are not yet in a form that is convenient for exploring large $\mu$ expansions. The large $\mu$ limit is of particular interest in light of the proposed correspondence [8] between type IIB superstring theory in the plane wave background and a certain sector of $\mathcal{N}=4 \mathrm{SU}(N)$ gauge theory, since the dual gauge theory is believed to be effectively perturbative in the parameter $\lambda^{\prime}=1 / \mu^{2}$. In the final section of this paper we present explicit formulas, which are valid to all orders in $\lambda^{\prime}$ perturbation theory, but omit non-perturbative terms of order $e^{-2 \pi \mu\left|\alpha_{r}\right|}$. The latter terms can in principle be extracted from our results with sufficient effort. We use these formulas to make a specific gauge theory prediction to all orders in $\lambda^{\prime}$.

\section{REVIEW OF BASIC FORMULAS}

The three-string interaction vertex for type IIB superstrings in flat space was worked out in $[9,10]$ and generalized to the plane-wave geometry in $[1,2]$. This vertex is represented as a state in the tensor product of three string Fock spaces, where the individual strings are labeled by an index $r=1,2,3$. Any particular three string coupling is then obtained by contracting it with three specific string states. The formula for the three-string interaction vertex contains a bosonic factor

$$
\left|V_{B}\right\rangle=\exp \left(\frac{1}{2} \sum_{r, s=1}^{3} \sum_{m, n=-\infty}^{\infty} \sum_{I=1}^{8} a_{r m}^{I \dagger} \bar{N}_{m n}^{r s} a_{s n}^{I \dagger}\right)|0\rangle .
$$

The quantities $\bar{N}_{m n}^{r s}$ are called Neumann coefficients. The three-string vertex also contains a similar fermionic factor $\left|V_{F}\right\rangle$ made out of the fermionic oscillators and a "prefactor" that is polynomial in the various oscillators. We will not discuss either of these in this paper. However, aside from an overall factor $v(\mu, y)$ that does not involve the oscillators, 
they are constructed out of essentially the same quantities, so the formulas that will be derived here determine them also.

In describing the Neumann matrices, it is convenient to consider separately the cases in which each of the indices $m, n$ are either positive, negative or zero. Henceforth, the symbols $m, n$ will always denote positive integers. One result of [1], for example, using matrix notation for the blocks with positive indices, is

$$
\bar{N}^{r s}=\delta^{r s}-2\left(C_{r} C^{-1}\right)^{1 / 2} A^{(r) \mathrm{T}} \Gamma_{+}^{-1} A^{(s)}\left(C_{s} C^{-1}\right)^{1 / 2} .
$$

Here $C_{m n}=m \delta_{m n}$ and $\left(C_{r}\right)_{m n}=\omega_{r m} \delta_{m n}$ are diagonal matrices, with

$$
\omega_{r m}=\sqrt{m^{2}+\left(\mu \alpha_{r}\right)^{2}}
$$

The definitions of the matrices $A^{(r)}$ and $\Gamma_{+}$will be given shortly.

The blocks with both indices negative are related in a simple way to the ones with both indices positive by

$$
\bar{N}_{-m-n}^{r s}=-\left(U_{r} \bar{N}^{r s} U_{s}\right)_{m n}
$$

where

$$
U_{r}=C^{-1}\left(C_{r}-\mu \alpha_{r}\right)=C\left(C_{r}+\mu \alpha_{r}\right)^{-1} .
$$

In the case of $\bar{N}^{33}$ these are the only nonvanishing terms. For the remaining Neumann coefficients the other nonvanishing terms are

$$
\begin{gathered}
\bar{N}_{m 0}^{r s}=\bar{N}_{0 m}^{s r}=\sqrt{2 \mu \alpha_{s}} \epsilon^{s t} \alpha_{t}\left[\left(C_{r} C^{-1}\right)^{1 / 2} A^{(r) \mathrm{T}} \Gamma_{+}^{-1} B\right]_{m}, \\
s, t \in\{1,2\}, r \in\{1,2,3\}, \\
\bar{N}_{00}^{r s}=(-1)^{r+s+1}(1+\mu \alpha k) \frac{\alpha}{\sqrt{\alpha_{r} \alpha_{s}}}, \quad r, s \in\{1,2\}, \\
\bar{N}_{00}^{3 r}=\bar{N}_{00}^{r 3}=-\sqrt{\alpha_{r}}, \quad r \in\{1,2\},
\end{gathered}
$$

where $\alpha=\alpha_{1} \alpha_{2} \alpha_{3}=-y(1-y)$, and $k=k(\mu, y)$ will be defined shortly.

The matrices $A^{(r)}(y)$ and the vector $B(y)$ do not depend on $\mu$, so they are the same as in flat space. Namely,

$$
\begin{gathered}
A_{m n}^{(1)}=\frac{2}{\pi}(-1)^{m+n+1} \sqrt{m n} \frac{y \sin m \pi y}{n^{2}-m^{2} y^{2}}, \\
A_{m n}^{(2)}=\frac{2}{\pi}(-1)^{m} \sqrt{m n} \frac{(1-y) \sin m \pi y}{n^{2}-m^{2}(1-y)^{2}}, \\
A_{m n}^{(3)}=\delta_{m n}, \\
B_{m}=\frac{2}{\pi}(-1)^{m+1} \frac{\sin m \pi y}{y(1-y) m^{3 / 2}} .
\end{gathered}
$$

A number of useful identities relating these matrices are included in Appendix A. Out of these and the diagonal matrices $U_{r}$ we construct

$$
\Gamma_{+}=\sum_{r=1}^{3} A^{(r)} U_{r} A^{(r) \mathrm{T}} .
$$

Note that the only $\mu$ dependence enters via $U_{r}$.

The quantities that we especially would like to evaluate explicitly are the matrix $\Gamma_{+}^{-1}(\mu, y)$, the vector

$$
Y_{m}(\mu, y)=\left(\Gamma_{+}^{-1}(\mu, y) B(y)\right)_{m},
$$

and the scalar

$$
k(\mu, y)=B^{\mathrm{T}} \Gamma_{+}^{-1} B
$$

In the case of flat space $(\mu=0)$ the results are known. Specifically, the Neumann matrices in Eq. (2) may be written as

$$
\bar{N}_{m n}^{r s}=-\frac{m n \alpha}{m \alpha_{s}+n \alpha_{r}} \bar{N}_{m}^{r} \bar{N}_{n}^{s} \text { for } \mu=0,
$$

where

$$
\bar{N}_{m}^{r}=\frac{\sqrt{m}}{\alpha_{r}} f_{m}\left(-\alpha_{r+1} / \alpha_{r}\right) e^{m \tau_{0} / \alpha_{r}} \quad \text { for } \mu=0,
$$

where $\alpha_{4}=\alpha_{1}$ is understood,

$$
f_{m}(\gamma)=\frac{\Gamma(m \gamma)}{m ! \Gamma(m \gamma+1-m)}
$$

and

$$
\tau_{0}=\sum_{r=1}^{3} \alpha_{r} \ln \left|\alpha_{r}\right|=y \ln y+(1-y) \ln (1-y) .
$$

In particular, still for $\mu=0, \Gamma_{+}^{-1}=\frac{1}{2}\left(1-\bar{N}^{33}\right), Y_{m}=-\bar{N}_{m}^{3}$, and $k=2 \tau_{0} / \alpha$. In other words,

$$
\begin{aligned}
Y_{m}(\mu & =0, y)=\frac{\sqrt{m} \Gamma(m y)}{m ! \Gamma(m y+1-m)} e^{-m \tau_{0}} \\
& =\frac{\Gamma(1+m y) \Gamma(1+m(1-y))}{2 \Gamma(1+m)} e^{-m \tau_{0}} B_{m}
\end{aligned}
$$

and

$$
k(\mu=0, y)=-2\left(\frac{\ln y}{1-y}+\frac{\ln (1-y)}{y}\right) .
$$

In $[4,5]$ the following identity was derived for arbitrary $\mu$ :

$$
\left\{\Gamma_{+}^{-1}, C_{3}\right\}=C+\frac{1}{2} \frac{\alpha_{1} \alpha_{2}}{1+\mu \alpha k} C U_{3}^{-1} Y Y^{\mathrm{T}} C U_{3}^{-1}
$$

Note that this determines $\Gamma_{+}^{-1}(\mu, y)$ in terms of $Y(\mu, y)$ and $k(\mu, y)$. In particular, this formula was shown to imply that the generalization of Eq. (11) to nonzero $\mu$ takes the form 


$$
\bar{N}_{m n}^{r s}=-\frac{m n \alpha}{1+\mu \alpha k} \frac{\bar{N}_{m}^{r} \bar{N}_{n}^{s}}{\alpha_{s} \omega_{r m}+\alpha_{r} \omega_{s n}}
$$

where

$$
\bar{N}_{m}^{r}=-\left[\left(C^{-1} C_{r}\right)^{1 / 2} U_{r}^{-1} A^{(r) \mathrm{T}} Y\right]_{m},
$$

but neither it nor $k(\mu, y)$ was determined explicitly at nonzero $\mu$.

Some preliminary analysis of large $\mu$ asymptotics was initiated in $[2-4,12]$, though not much can be done without additional explicit formulas. It was found that the leading (large $\mu$ ) term in the expansion of $\Gamma_{+}^{-1}$ is given by the first term on the right-hand side of Eq. (17). Defining $R$ by

$$
\Gamma_{+}^{-1}=\frac{1}{2} C C_{3}^{-1}+R
$$

it is easy to see that the leading term in $R$ is of order $\mu^{-4}$. Specifically,

$$
R \rightarrow a_{R} \pi \frac{y^{2}(1-y)^{2}}{\mu^{4}} C^{3} B B^{\mathrm{T}} C^{3}+\cdots
$$

where $a_{R}$ is a constant and the next term in the expansion is of order $\mu^{-6}$. Similarly,

$$
k(\mu, y) \rightarrow \frac{1}{\mu y(1-y)}-\frac{a_{k}}{\pi[\mu y(1-y)]^{2}}+\cdots .
$$

Inserting these expansions into Eq. (17), one learns that

$$
a_{R} a_{k}=\frac{1}{64} \text {. }
$$

It is very difficult to determine $a_{R}$ and $a_{k}$ separately without additional explicit formulas. The asymptotic expansion of $Y$ was found to have the structure

$$
Y_{m} \rightarrow \frac{1}{\mu}\left[\frac{1}{2} m-\left(\frac{1}{4}-x\right) \frac{m^{3}}{\mu^{2}}+\cdots\right] B_{m} .
$$

The value of $x$ is of particular interest. It was estimated numerically to be approximately $1 / 16$ in [3], and we will show below that this is correct.

\section{THE DIFFERENTIAL EQUATION}

This section describes the derivation of a differential equation involving $Y_{m}(\mu, y)$ and $k(\mu, y)$. For the benefit of the reader who would like to skip the details of the derivation, and move on to the next section, the result is stated here:

$$
\frac{\partial Y_{m}}{\partial \mu}=\left[\frac{1}{2} \frac{\partial F}{\partial \mu}\left(1-\frac{\mu}{\omega_{m}}\right)-\frac{\mu}{\omega_{m}^{2}}\right] Y_{m}
$$

where $\omega_{m}=\omega_{3 m}=\sqrt{m^{2}+\mu^{2}}$ and

$$
F(\mu, y)=\ln [1+\mu \alpha k(\mu, y)]=\ln [1-\mu y(1-y) k(\mu, y)] .
$$

This has the formal solution

$$
Y_{m}(\mu, y)=\frac{m}{\omega_{m}} \exp \left[\frac{1}{2} \int_{0}^{\mu} \frac{\partial F}{\partial \mu}\left(1-\frac{\mu}{\omega_{m}}\right) d \mu\right] Y_{m}(0, y) .
$$

Thus, if we knew $k(\mu, y)$, we would know $F(\mu, y)$, and then $Y_{m}(\mu, y)$, and hence all the Neumann coefficients.

The derivation of Eq. (25) is rather involved. Let us sketch the derivation here and then fill in some of the details in Appendix B. The matrix $\Gamma_{+}=\sum A^{(r)} U_{r} A^{(r) \mathrm{T}}$ only depends on $\mu$ through the dependence of $U_{r}$ on $\mu$. Its derivative can be written in the form

$$
\frac{\partial \Gamma_{+}}{\partial \mu}=-\frac{1}{2} \alpha B B^{\mathrm{T}}+\mu N,
$$

where

$$
N=\sum_{r=1}^{3} \alpha_{r}^{2} A^{(r)} C^{-1} C_{r}^{-1} A^{(r) \mathrm{T}}
$$

It follows that

$$
\frac{\partial Y}{\partial \mu}=\frac{1}{2} k \alpha Y-\mu \Gamma_{+}^{-1} N Y .
$$

But $N Y$ can be recast in the form

$$
N Y=g_{1} C_{3}^{-2} B+g_{2} B,
$$

where the coefficients $g_{1}$ and $g_{2}$ are scalar quantities:

$$
\begin{aligned}
& g_{1}=\frac{2(1+\mu \alpha k)}{2+\mu \alpha k+\mu^{2} \alpha k_{1}}, \\
& g_{2}=\left(\frac{\alpha}{2}\right) \frac{\alpha k^{2}+\mu \alpha k k_{1}+2 k_{1}}{2+\mu \alpha k+\mu^{2} \alpha k_{1}},
\end{aligned}
$$

and

$$
k_{i}=B^{\mathrm{T}} C_{3}^{-i} Y
$$

The above equations imply that

$$
\frac{\partial k}{\partial \mu}=B^{\mathrm{T}} \frac{\partial Y}{\partial \mu}=\frac{1}{2} \alpha k^{2}-\mu g_{2} k-\mu g_{1} k_{2} .
$$

This is not very useful as it stands, since there is no other apparent way to determine $k_{2}$. ( $k_{1}$ could be determined, but that will turn out not to be necessary.) Substituting the equation for $N Y$ and an identity for $\left[C_{3}^{-2}, \Gamma_{+}^{-1}\right]$ deduced from Eq. (17), one can recast the derivative of $Y$ in the form

$$
\frac{\partial Y}{\partial \mu}=\left(F_{0}+F_{1} C_{3}^{-1}+F_{2} C_{3}^{-2}\right) Y,
$$


where the scalar functions $F_{i}$ are given by

$$
\begin{aligned}
& F_{0}=\frac{1}{2} \alpha k-\mu g_{2}+\frac{1}{2} \mu g_{1} \frac{\alpha}{1+\mu \alpha k}\left(k_{1}-\mu k_{2}\right), \\
& F_{1}=-\frac{1}{2} \mu g_{1} \frac{\alpha}{1+\mu \alpha k}\left(k-\mu^{2} k_{2}\right), \\
& F_{2}=-\mu g_{1}+\frac{1}{2} \mu^{2} g_{1} \frac{\alpha}{1+\mu \alpha k}\left(k-\mu k_{1}\right) .
\end{aligned}
$$

Using Eq. (32) for $g_{1}$ and $g_{2}$, and Eq. (34) to eliminate $k_{2}$ in favor of $k^{\prime}$, we find

$$
F_{2}=-\mu, \quad F_{1}=-\mu F_{0}, \quad F_{0}=\frac{\alpha}{2} \frac{1}{1+\mu \alpha k}\left(k+\mu k^{\prime}\right),
$$

so that Eq. (35) can be written in the form (25).

As a first application of Eq. (25), let us consider it for large $\mu$. The expansion in Eq. (22) implies that at large $\mu$ the leading behavior of $\partial F / \partial \mu$ is $-1 / \mu$. Substituting this into Eq. (24), one deduces that $x=1 / 16$.

\section{AN INTEGRAL TRANSFORM}

In this section we show that the known large $\mu$ behavior $Y_{m} \sim(m / 2 \mu) B_{m}$, together with Eq. (27), is sufficient to deduce an integral transform, whose solution we are able to determine explicitly, thereby obtaining explicit formulas for $k(\mu, y), F(\mu, y)$ and hence $Y_{m}(\mu, y)$.

Since $Y_{m} \sim(m / 2 \mu) B_{m}$ for large $\mu$, Eq. (27) implies that

$$
\exp \left\{\frac{1}{2} \int_{0}^{\infty} \frac{\partial F}{\partial \mu}\left(1-\frac{\mu}{\omega_{m}}\right) d \mu\right\}=\frac{B_{m}}{2 Y_{m}(0)} .
$$

Taking the logarithm of both sides and integrating by parts, this becomes

$$
\int_{0}^{\infty}\left(m^{2}+\mu^{2}\right)^{-3 / 2} F(\mu, y) d \mu=G(m, y),
$$

where, using Eq. (15),

$$
G(z, y)=\frac{2 \tau_{0}}{z}+\frac{2}{z^{2}} \ln \left(\frac{\Gamma(1+z)}{\Gamma(1+z y) \Gamma(1+z(1-y))}\right)
$$

Equation (39), which must hold for $m=1,2, \ldots$, and $0 \leqslant y$ $\leqslant 1$, determines $F(\mu, y)$ and hence $k(\mu, y)$.

The function $G(z, y)$ is holomorphic in the right-half $z$ plane and goes to 0 as $z \rightarrow \infty$ in that half plane like a power $\left(1 / z^{2}\right)$. These properties make this extrapolation from the positive integers to a continuous variable $z$ unique. Moreover, they are exactly what is needed to construct an inverse transformation giving $F$ in terms of $G$. Let us state this as:

Theorem. Suppose that $g(z)$ is holomorphic in the righthalf $z$ plane and vanishes like a power at infinity in that half plane. Then the solution of the equation

$$
\int_{0}^{\infty}\left(z^{2}+x^{2}\right)^{-3 / 2} f(x) d x=g(z)
$$

[for $\operatorname{Re}(z)>0$ ] is given by the inverse integral transform

$$
\begin{aligned}
f(x) & =-i \frac{x^{2}}{\pi} \int_{0}^{\pi} g(-i x \cos \theta) \cos \theta d \theta \\
& =-i \frac{x^{2}}{\pi} \int_{0}^{\pi / 2}[g(-i x \cos \theta)-g(i x \cos \theta)] \cos \theta d \theta .
\end{aligned}
$$

We refer the reader to Appendix $\mathrm{C}$ for the proof of this theorem. that

Applying the theorem to the problem at hand, we learn

$$
F(\mu, y)=-i \frac{\mu^{2}}{\pi} \int_{0}^{\pi} G(-i \mu \cos \theta, y) \cos \theta d \theta .
$$

Substituting the formula for $G$,

$$
\begin{aligned}
F(\mu, y)= & 2 \mu \tau_{0}+\frac{2 i}{\pi} \int_{0}^{\pi} \frac{d \theta}{\cos \theta} \\
& \times \ln \left[\frac{\Gamma(1-i \mu \cos \theta)}{\Gamma(1-i \mu y \cos \theta) \Gamma(1-i \mu(1-y) \cos \theta)}\right]
\end{aligned}
$$

The formula for $Y_{m}$ also requires the derivative

$$
\begin{aligned}
\frac{\partial F(\mu, y)}{\partial \mu}= & 2 \tau_{0}+\frac{2}{\pi} \int_{0}^{\pi} d \theta[\psi(1-i \mu \cos \theta)-y \psi(1-i \mu y \\
& \times \cos \theta)-(1-y) \psi(1-i \mu(1-y) \cos \theta)]
\end{aligned}
$$

where, as usual, $\psi=(\ln \Gamma)^{\prime}$.

Substituting the expansion

$$
\psi(1+z)=-\gamma+z \sum_{n=1}^{\infty} \frac{1}{n(z+n)}
$$

and using the integral

$$
\int_{0}^{\pi} \frac{d \theta}{a+b \cos \theta}=\frac{\pi}{\sqrt{a^{2}-b^{2}}}
$$

gives the result

$$
\frac{\partial F(\mu, y)}{\partial \mu}=2 \tau_{0}+2 \sum_{n=1}^{\infty} \sum_{r=1}^{3} \frac{\alpha_{r}}{\omega_{r n}} .
$$


Since $F(0, y)=0$, this integrates to

$$
\begin{aligned}
F(\mu, y)= & 2 \tau_{0} \mu+2 \sum_{n, r} \ln \left[\left(\omega_{r n}+\mu \alpha_{r}\right) / n\right]=2 \tau_{0} \mu \\
& -2 \ln \operatorname{det}\left(U_{1} U_{2} U_{3}\right) .
\end{aligned}
$$

Note that $\operatorname{det}\left(U_{1} U_{2} U_{3}\right)$ is convergent even though the individual $\operatorname{det}\left(U_{r}\right)$ diverge. We can regulate them in a way that does not change the product (since $\Sigma \alpha_{r}=0$ ) by recasting $F$ in the form

$$
F(\mu, y)=2 \tau_{0} \mu+2 \sum_{r} \phi\left(\mu \alpha_{r}\right),
$$

where

$\phi(x)=\sum_{n=1}^{\infty}\left[\ln \left(\frac{\sqrt{n^{2}+x^{2}}+x}{n}\right)-\frac{x}{n}\right]=\sum_{n=1}^{\infty}\left[\operatorname{arcsinh}\left(\frac{x}{n}\right)-\frac{x}{n}\right]$.

The regulated determinant of $U_{r}$ is then $\exp \left[-\phi\left(\mu \alpha_{r}\right)\right]$. Substituting the expansion of $\partial F / \partial \mu$ into the formula for $Y_{m}$ gives

$$
Y_{m}(\mu, y)=\exp \left[\left(\mu-\omega_{m}\right) \tau_{0}+\sum_{r=1}^{3}\left(\phi_{r}-\phi_{m r}\right)\right] \frac{m}{2 \omega_{m}} B_{m},
$$

where $\phi_{r}=\phi\left(\mu \alpha_{r}\right)$ and

$$
\phi_{m r}=\sum_{n=1}^{\infty}\left[\ln \left(\frac{\omega_{r n}+\omega_{m} \alpha_{r}}{n}\right)-\frac{\omega_{m} \alpha_{r}}{n}\right],
$$

where, we remind the reader, $\omega_{m}=\sqrt{m^{2}+\mu^{2}}$ and $\omega_{r m}$ $=\sqrt{m^{2}+\left(\mu \alpha_{r}\right)^{2}}$.

The formulas (50) and (52) are explicit expansions of $F$ and $Y_{m}$ which converge for all finite $\mu$. Thus, in a sense, they solve our problem. However, they are not yet in the most convenient form for exploring large $\mu$ expansions.

\section{ASYMPTOTIC EXPANSIONS FOR LARGE $\mu$}

In this section we develop asymptotic large $\mu$ expansions for all quantities that appear in the three-string vertex. It turns out that the quantities all have essential singularities at $\mu=\infty$ arising from terms proportional to $e^{-2 \pi \mu\left|\alpha_{r}\right|}$. The existence of such terms, which correspond to non-perturbative effects in the dual gauge theory, has been noted in [3]. We proceed under the assumption that $\mu\left|\alpha_{r}\right|$ is sufficiently large (for all $r$ ) that these terms can be neglected, and we use the symbol $\approx$ to denote this approximation. We stress that the formulas we present encapsulate all orders in a power series expansion around $\lambda^{\prime}=0$ from the dual gauge theory point of view. However, they do not match smoothly with the known flat space results at $\mu=0$ because the omitted terms become important in this limit. Finally, the limit $y \rightarrow 0$ (at fixed $\mu$ ) is also of interest, because it can be used to extract vertex operators for the emission of on-shell particles [9]. Our asymptotic formulas are not well-suited for studying this limit because we omit terms of order $e^{-2 \pi \mu y}$.

Let us demonstrate how to use the integral equation (39) directly to find the constant $a_{k}$ introduced in Eq. (22). It follows from Eq. (22) that

$$
F(\mu, y)=-\ln \left[\pi \mu y(1-y) / a_{k}\right]+\cdots
$$

at large $\mu$. Let us define $\widetilde{F}(\mu, y)=F(\mu, y)+\ln [\pi \mu y(1$ $\left.-y) / a_{k}\right]$. Then Eq. (39) implies that

$$
\begin{aligned}
\int_{0}^{\infty}\left(m^{2}+\mu^{2}\right)^{-3 / 2} \widetilde{F}(\mu, y) d \mu \\
\quad=G(m, y)+\frac{1}{m^{2}} \ln \left(\frac{m \pi y(1-y)}{2 a_{k}}\right) .
\end{aligned}
$$

Now let $m=n \lambda$ and $\mu=\lambda w$. After scaling out $\lambda$, we find

$$
\begin{aligned}
\int_{0}^{\infty}\left(w^{2}+n^{2}\right)^{-3 / 2} \widetilde{F}(\lambda w, y) d w \\
\quad=\lambda^{2}\left[G(\lambda n, y)+\frac{1}{\lambda^{2} n^{2}} \ln \left(\frac{\lambda n \pi y(1-y)}{2 a_{k}}\right)\right] .
\end{aligned}
$$

In the limit $\lambda \rightarrow \infty$ the left-hand side goes to zero, while from Eq. (40) it is easy to check using Stirling's approximation that the right-hand side goes to $\left(1 / n^{2}\right) \ln \left(4 a_{k}\right)$. This determines $a_{k}=1 / 4$ and, from Eq. (23), $a_{R}=\frac{1}{16}$.

\section{A. $F(\mu, y)$ and $k(\mu, y)$}

Let us proceed by studying the function $\phi(x)$ defined in Eq. (51). Since $\phi$ is clearly odd, it is sufficient to consider large positive $x$ here. Taking two derivatives gives

$$
\phi^{\prime \prime}(x)=-x \sum_{n=1}^{\infty} \frac{1}{\left(x^{2}+n^{2}\right)^{3 / 2}} \approx-\frac{1}{x}+\frac{1}{2 x^{2}},
$$

where we have used Eq. (E5) to evaluate the sum [the exact result is given in Eq. (F4)]. Integrating twice with respect to $x$ leads to

$$
\phi(x) \approx-\left(x+\frac{1}{2}\right) \ln x+c_{1} x+c_{2},
$$

where $c_{i}$ are constants of integration. Inserting Eq. (58) into Eq. (50) gives

$$
F(\mu, y) \approx-\ln [\mu y(1-y)]+2 c_{2} .
$$

The constant $c_{1}$ has dropped out since $\sum \alpha_{r}=0$, and we can determine $c_{2}=\frac{1}{2} \ln 4 \pi$ by comparing Eq. (59) to Eq. (54) with $a_{k}=\frac{1}{4}$, obtaining thereby

$$
F(\mu, y) \approx-\ln [4 \pi \mu y(1-y)] .
$$

We emphasize that Eq. (60) is much stronger than Eq. (54): when we wrote the latter, we might have expected corrections involving powers of $1 / \mu$, but in Eq. (60) we have proven that the only corrections are exponentially small. An 
alternative derivation of this result is presented in Appendix $\mathrm{D}$, where we show that the absence of power law corrections follows from a simple contour integral argument. In Appendix $\mathrm{F}$ we derive the exact formula

$$
\begin{aligned}
F(\mu, y)= & -\ln [4 \pi \mu y(1-y)]+J(\mu y)+J(\mu(1-y)) \\
& -J(\mu)
\end{aligned}
$$

where

$$
J(x)=\frac{2}{\pi} \int_{1}^{\infty} \frac{\ln \left(1-e^{-2 \pi x z}\right)}{z \sqrt{z^{2}-1}} d z
$$

It is easy to read off all the exponential corrections to Eq. (61) by writing out the series expansion of the logarithm. However, we will not keep track of these exponential corrections in the following sections, and instead simply use Eq. (60) and the definition (26) to write

$$
\begin{gathered}
k(\mu, y) \approx \frac{1}{\mu y(1-y)}-\frac{1}{4 \pi \mu^{2} y^{2}(1-y)^{2}} . \\
\text { B. } \boldsymbol{Y}_{\boldsymbol{m}}(\boldsymbol{\mu}, \boldsymbol{y})
\end{gathered}
$$

Although it is straightforward to develop an asymptotic expansion for the function $\phi_{m r}$ defined in Eq. (53), a more direct route is simply to rewrite Eq. (27) as

$$
\begin{aligned}
Y_{m}(\mu, y)= & \frac{m}{\omega_{m}} \exp \left[\frac{1}{2} \int_{0}^{\infty} \frac{\partial F}{\partial \mu}\left(1-\frac{\mu}{\omega_{m}}\right) d \mu\right. \\
& \left.-\int_{\mu}^{\infty} \frac{\partial F}{\partial \mu}\left(1-\frac{\mu}{\omega_{m}}\right) d \mu\right] Y_{m}(0, y) .
\end{aligned}
$$

The first integral is just Eq. (38), and the second integral is elementary after substituting the asymptotic expansion $F^{\prime}$ $\approx-1 / \mu$. The final result is

$$
\begin{aligned}
Y_{m}(\mu, y) & \approx \sqrt{\frac{\mu+\omega_{m}}{2 \mu} \frac{m}{2 \omega_{m}} B_{m}} \\
& =\frac{1}{2 \sqrt{2 \mu}} \frac{\sqrt{\mu+\sqrt{m^{2}+\mu^{2}}}}{\sqrt{m^{2}+\mu^{2}}} m B_{m},
\end{aligned}
$$

which can be conveniently summarized as

$$
Y \approx \frac{1}{2 \sqrt{2 \mu}} U_{3}^{1 / 2} C^{3 / 2} C_{3}^{-1} B
$$

\section{The Neumann vectors}

The final step in the construction of the Neumann matrices involves evaluating the matrix products $A^{(r) \mathrm{T}} Y$ which appear in the Neumann vectors in Eq. (19). From Eq. (65) we have
$\left(A^{(1) \mathrm{T}} Y\right)_{n}$

$$
\approx \sqrt{\frac{2}{\mu}} \frac{(-1)^{n+1} \sqrt{n}}{\pi^{2} y^{2}(1-y)} \sum_{m=1}^{\infty} \frac{\sin ^{2}(\pi m y)}{m^{2}-n^{2} / y^{2}} \frac{\sqrt{\mu+\sqrt{\mu^{2}+m^{2}}}}{\sqrt{\mu^{2}+m^{2}}} .
$$

Using Eq. (E9), we find

$$
\begin{aligned}
\left(A^{(r) \mathrm{T}} Y\right)_{n} \approx & \frac{1}{\pi y(1-y) 2 \sqrt{2 \mu}} \\
& \times(-1)^{r(n+1)-1} \sqrt{\alpha_{r}} \omega_{r n}^{-1} U_{r n}^{1 / 2}, \quad r \in\{1,2\} .
\end{aligned}
$$

The Neumann vectors in Eq. (19) can therefore be expressed as

$$
\begin{aligned}
& \bar{N}_{n}^{r} \approx \frac{1}{2 \pi y(1-y)}(-1)^{r(n+1)} \sqrt{\alpha_{r}}\left(2 \mu n \omega_{r n} U_{r n}\right)^{-1 / 2}, \\
& r \in\{1,2\}, \\
& \bar{N}_{n}^{3} \approx-\frac{n}{2}\left(2 \mu \omega_{3 n} U_{3 n}\right)^{-1 / 2} B_{n} .
\end{aligned}
$$

Actually we can combine these expressions in a useful way. If we define

$$
s_{1 m}=s_{2 m}=1, \quad s_{3 m}=-2 \sin (\pi m y),
$$

then we have simply

$$
\bar{N}_{n}^{r} \approx \frac{(-1)^{r(n+1)}}{2 \pi y(1-y)} \sqrt{\frac{\left|\alpha_{r}\right|}{2 \mu n \omega_{r n} U_{r n}}} s_{r n}, \quad r \in\{1,2,3\} .
$$

\section{Consistency checks}

We should check that the vector $Y$ we have found indeed satisfies $B^{\mathrm{T}} Y \approx k$ and $\Gamma_{+} Y \approx B$. First we have

$$
\begin{aligned}
B^{\mathrm{T}} Y \approx & \frac{4}{\pi^{2}} \frac{1}{y^{2}(1-y)^{2}} \frac{1}{2 \sqrt{2 \mu}} \\
& \times \sum_{m=1}^{\infty} \frac{\sin ^{2}(\pi m y)}{m^{2}} \frac{\sqrt{\mu+\sqrt{\mu^{2}+m^{2}}}}{\sqrt{\mu^{2}+m^{2}}} .
\end{aligned}
$$

With the help of Eq. (E10), it immediately follows that this expression equals Eq. (63). 
In [3] it was shown that $\Gamma_{+}=2 C_{3} C^{-1}-H$, where $H$ is given up to exponential corrections by

$$
\begin{aligned}
H_{m n} \approx & \frac{8}{\mu^{2} \pi^{2}}(-1)^{m+n} \sqrt{m n} \sin (\pi m y) \sin (\pi n y) \\
& \times \int_{1}^{\infty} d z \frac{\sqrt{z^{2}-1}}{\left(z^{2}+m^{2} / \mu^{2}\right)\left(z^{2}+n^{2} / \mu^{2}\right)} .
\end{aligned}
$$

[The integral is easily evaluated, but it is convenient to leave Eq. (73) in this form for the calculation.] The condition $\Gamma_{+} Y=B$ which we would now like to check is equivalent to

$$
H Y=2 C_{3} C^{-1} Y-B \text {. }
$$

Using Eqs. (73) and (65) we can write

$$
\begin{aligned}
(H Y)_{n} \approx & \frac{2 \sqrt{2} n^{2} B_{n}}{\pi^{2} \sqrt{\mu}} \int_{1}^{\infty} \frac{\sqrt{z^{2}-1}}{z^{2}+n^{2} / \mu^{2}} \\
& \times \sum_{m=1}^{\infty} \frac{\sin (\pi m y)^{2}}{m^{2}+z^{2} \mu^{2}} \frac{\sqrt{\mu+\sqrt{\mu^{2}+m^{2}}}}{\sqrt{m^{2}+\mu^{2}}} .
\end{aligned}
$$

After substituting Eq. (E11) for the sum, the remaining integral over $z$ is elementary and takes the form

$$
\begin{aligned}
& \operatorname{Re} \int_{1}^{\infty} d z \frac{\sqrt{-1+i \sqrt{z^{2}-1}}}{z\left(z^{2}+a^{2}\right)} \\
& \quad=-\frac{\pi}{\sqrt{2} a^{2}}\left[1-\frac{1}{\sqrt{2}} \sqrt{1+\sqrt{1+a^{2}}}\right]
\end{aligned}
$$

for $a=n / \mu$. Assembling all factors from Eqs. (75), (76) and (E11), we find

$$
(H Y)_{n} \approx-B_{n}\left[1-\frac{1}{\sqrt{2}} \sqrt{1+\sqrt{1+n^{2} / \mu^{2}}}\right] .
$$

Recalling Eq. (65), we see that the desired relation Eq. (74) is indeed satisfied up to exponential corrections.

\section{E. Some remaining quantities}

The Neumann matrices are completely determined, to all orders in $1 / \mu$, by the factorization identity (18) and the Neumann vectors given in Eq. (71). For the sake of completeness, we catalog here two more quantities of interest, which follow easily from our results. The first is the matrix $R$ defined in Eq. (20). By comparing the expressions (2) and (18) for $r=s=3$ we can determine $\Gamma_{+}^{-1}$, and hence $R$, in terms of $Y_{m}$. Using Eq. (66), we arrive at

$$
\begin{aligned}
R_{m n} & =\left(\Gamma_{+}^{-1}\right)_{m n}-\frac{1}{2} \frac{m}{\sqrt{m^{2}+\mu^{2}}} \delta_{m n} \\
& \approx \frac{1}{\pi} m n(-1)^{m+n} \frac{\sin (\pi m y) \sin (\pi n y)}{\omega_{n} \omega_{m}\left(\omega_{m}+\omega_{n}\right) \sqrt{U_{m} U_{n}}},
\end{aligned}
$$

where (as before)

$$
\omega_{m}=\sqrt{m^{2}+\mu^{2}}, \quad U_{m}=\frac{\omega_{m}+\mu}{m} .
$$

Finally, we remarked above that the prefactor is polynomial in quantities $X$ and $Y$ which are constructed out of bosonic and fermionic oscillators respectively (see [2,5,7]). The normalization of $X$ and $Y$ involves a factor called $f(\mu)$ in [5]. It may be obtained from the formula [2,5]

$$
f(\mu)=\sqrt{-2 \pi \alpha_{2} \alpha_{3}} \lim _{n \rightarrow \infty}(-1)^{n}\left(C A^{(1) \mathrm{T}} \Gamma_{+}^{-1} B\right)_{n} .
$$

It follows immediately from Eq. (68) that

$$
f(\mu) \approx \frac{1}{\sqrt{4 \pi \mu y(1-y)}} \approx \sqrt{1+\mu \alpha k} .
$$

In fact, closure of the supersymmetry algebra requires $f(\mu)$ $=\sqrt{1+\mu \alpha k}$ [7]. Note that this $f(\mu)$ is separate from a still undetermined function $v(\mu)$, to appear below, which appears as an overall factor in the cubic part of the Hamiltonian and supersymmetry generators.

\section{F. Summary of Neumann matrices}

We summarize here the final expressions for the Neumann matrices obtained in this paper. As before, we use the notation

$$
\begin{gathered}
\alpha_{1}=y, \quad \alpha_{2}=1-y, \quad \alpha_{3}=-1, \\
s_{1 m}=s_{2 m}=1, \quad s_{3 m}=-2 \sin (\pi m y),
\end{gathered}
$$

and $\omega_{r m}=\sqrt{m^{2}+\left(\mu \alpha_{r}\right)^{2}}$. Then for $m, n>0$ we have

$$
\begin{aligned}
\bar{N}_{m n}^{r s} \approx & \frac{1}{2 \pi} \frac{(-1)^{r(m+1)+s(n+1)}}{\alpha_{s} \omega_{r m}+\alpha_{r} \omega_{s n}} \\
& \times \sqrt{\frac{\left|\alpha_{r} \alpha_{s}\right|\left(\omega_{r m}+\mu \alpha_{r}\right)\left(\omega_{s n}+\mu \alpha_{s}\right)}{\omega_{r m} \omega_{s n}}} s_{r m} s_{s n},
\end{aligned}
$$

$$
\begin{aligned}
\bar{N}_{-m,-n}^{r s} \approx & -\frac{1}{2 \pi} \frac{(-1)^{r(m+1)+s(n+1)}}{\alpha_{s} \omega_{r m}+\alpha_{r} \omega_{s n}} \\
& \times \sqrt{\frac{\left|\alpha_{r} \alpha_{s}\right|\left(\omega_{r m}-\mu \alpha_{r}\right)\left(\omega_{s n}-\mu \alpha_{s}\right)}{\omega_{r m} \omega_{s n}}} s_{r m} s_{s n} .
\end{aligned}
$$

As before, the symbol $\approx$ denotes that we have omitted terms of order $e^{-2 \pi \mu\left|\alpha_{r}\right|}$ (for all $r$ ). For $r=s=3$ these are the only nonzero components. If we define

$$
s_{10}=s_{20}=\frac{1}{\sqrt{2}}, \quad s_{30}=0,
$$

then Eq. (84) continues to hold when $m$ is zero and $n$ is positive (or vice versa). Finally, if $n=m=0$ we have 


$$
\begin{gathered}
\bar{N}_{00}^{r s} \approx \frac{1}{4 \pi \mu} \frac{(-1)^{r+s}}{\sqrt{\alpha_{r} \alpha_{s}}}, \quad r, s \in\{1,2\}, \\
\bar{N}_{00}^{3 r}=\bar{N}_{00}^{r 3}=-\sqrt{\alpha_{r}}, \quad r \in\{1,2\} .
\end{gathered}
$$

We remark here that we have been assuming throughout this work that $\mu$ is positive, since it is clear from the analysis of [1] that only the absolute value of $\mu$ enters into the bosonic matrix elements. The behavior of various quantities under $\mu \rightarrow-\mu$ was exploited in [4] to derive several useful identities. Although we have not considered this involution here, it may be worthwhile to do so.

\section{A MATRIX ELEMENT}

In this section we use our result to calculate, to all orders in $\lambda^{\prime}$, a particular matrix element of the Hamiltonian which has so far only been computed to first order in $\lambda^{\prime}$ in the dual gauge theory $[6,15,19]$. We then explain in detail how this matrix element is encoded in the gauge theory.

\section{A. String field theory}

Consider for $m, n>0$ the three states ${ }^{1}$

$$
\begin{aligned}
& \langle 1|=\frac{10}{1}\langle 0|\left(a_{m}^{i}-i a_{-m}^{i}\right)\left(a_{m}^{j}+i a_{-m}^{j}\right), \\
& \langle 2|=\langle 0|, \\
& \langle 3|=\frac{10}{1}\langle 0|\left(a_{n}^{i}-i a_{-n}^{i}\right)\left(a_{n}^{j}+i a_{-n}^{j}\right),
\end{aligned}
$$

where $i$ and $j$ are $\mathrm{SO}(4)$ indices. The two-impurity states $\langle 1|$ and $\langle 3|$ decompose into the $\mathbf{1}, \mathbf{6}$ and $\mathbf{9}$ representations of $\mathrm{SO}(4)$. For definiteness, we fix $i \neq j$, and we could choose to symmetrize or antisymmetrize in $i j$ at the end of the calculation. Actually, it turns out that the matrix element vanishes when either two-impurity state is in the $\mathbf{6}$.

We have not discussed the prefactor in this paper, but it has been shown in $[2,6]$ that for states of the form (89) (in particular, for states with no fermionic excitations), the threestring coupling $|H\rangle$ in the Hamiltonian is given effectively by

$$
|H\rangle=v(\mu, y) \mathcal{P}\left|V_{B}\right\rangle,
$$

where $\left|V_{B}\right\rangle$ was defined in Eq. (1) and we have defined ${ }^{2}$

$$
\begin{aligned}
\mathcal{P} & =\frac{\alpha}{2} \sum_{r=1}^{3}\left[\sum_{m=-\infty}^{\infty} \frac{\omega_{r m}}{\mu \alpha_{r}} e(m) a_{r m}^{\dagger} a_{r m}\right], \\
e(m) & =\left\{\begin{array}{cl}
1 & m \geqslant 0, \\
-1 & m<0,
\end{array}\right.
\end{aligned}
$$

\footnotetext{
${ }^{1}$ We caution the reader that the basis of oscillators employed here and in $[1,2]$ differs from that used by [8] and most gauge theory papers by the transformation $a_{n}^{\mathrm{BMN}}=(1 / \sqrt{2})\left(a_{|n|}-i \operatorname{sgn}(n) a_{-|n|}\right)$ for $n \neq 0$.

${ }^{2}$ The apparent discrepancy between this formula and the one given in [6] is entirely due to the change of basis in footnote 1 .
}

where the $\mathrm{SO}(4)$ index (which we have suppressed) is contracted between $a^{\dagger}$ and $a$. Finally, the function $v(\mu, y)$ is a measure factor which has not yet been determined. It arises from the path integral which defines the cubic string vertex. In flat space, the function can be determined by Lorentz invariance (it is 1 in the supersymmetric theory, and some very complicated function of $y$ in the bosonic theory). The plane wave superalgebra does not have enough generators to fix this overall factor, although comparison with gauge theory requires that $v(\mu, y) \rightarrow 1$ for large $\mu$. The corresponding factor in the supergravity vertex in the plane wave background has been determined to be a constant [11].

For the states (89) we find the matrix element

$$
\begin{aligned}
H_{n m y} \equiv & \langle 1|\langle 2|\langle 3 \mid H\rangle=v(\mu, y) \frac{1}{4} \frac{\alpha}{2}\left(\frac{\omega_{1 m}}{\mu \alpha_{1}}+\frac{\omega_{3 n}}{\mu \alpha_{3}}\right)\left[2\left(\bar{N}_{m n}^{13}\right)^{2}\right. \\
& \left.-2\left(\bar{N}_{-m,-n}^{13}\right)^{2}\right] .
\end{aligned}
$$

Using Eqs. (84) and (85), we find after dramatic cancellation the simple result

$$
H_{n m y} \approx \frac{1}{2 \mu^{2}}(1-y) \frac{\sin ^{2}(\pi n y)}{\pi^{2}}\left[\frac{v(\mu, y) \mu^{2} y}{\omega_{1 m} \omega_{3 n}}\right] .
$$

The quantity in brackets is equal to 1 at leading order for large $\mu$, reproducing the result presented in [6]. As is now standard in the literature, it should be understood that the cubic interaction $H$ considered here enters the full Hamiltonian with a coefficient equal to the effective string coupling, $g_{2}=4 \pi g_{s} \mu^{2}$.

\section{B. Relation to gauge theory}

The result (93) provides a concrete all-loop prediction for the gauge theory, which we now explain. Consider (in the large $J$ limit) the normalized Berenstein-Maldacena-Nastase (BMN) [8,14-16] operators

$$
\begin{aligned}
O_{n}= & \frac{1}{\sqrt{J N^{J+2}}} \sum_{k=0}^{J} e^{2 \pi i k n / J} \operatorname{Tr}\left(\phi^{i} Z^{k} \phi^{j} Z^{J-k}\right), \\
T_{n}^{y}= & \frac{1}{\sqrt{J y(1-y)}} \frac{1}{\sqrt{J N^{J+2}}} \\
& \times \sum_{k=0}^{J y} e^{2 \pi i k n y / J}: \operatorname{Tr}\left(\phi^{i} Z^{k} \phi^{j} Z^{J y-k}\right) \operatorname{Tr}\left(Z^{(1-y) J}\right): \\
T^{y}= & \frac{1}{\sqrt{N^{J+2}}}: \operatorname{Tr}\left(\phi^{i} Z^{(1-y) J}\right) \operatorname{Tr}\left(\phi^{j} Z^{y J}\right):
\end{aligned}
$$

where $i \neq j \in\{1,2,3,4\}$ are $\mathrm{SO}(4)$ indices [see the discussion below Eq. (89)] labelling four of the six scalar fields of $\mathcal{N}$ $=4 \mathrm{SU}(\mathrm{N})$ gauge theory, and $Z=(1 / \sqrt{2})\left(\phi^{5}+i \phi^{6}\right)$.

At zero string coupling $\left(g_{2}=0\right)$, the single- (double-) trace operators $O(T)$ defined in Eq. (94) correspond respectively to one- (two-) string states and have definite conformal dimensions 


$$
\Delta_{n}=J+2 \sqrt{1+\lambda^{\prime} n^{2}}, \quad \Delta_{n}^{y}=J+2 \sqrt{1+\lambda^{\prime} n^{2} / y^{2}}, \quad \Delta^{y}=J+2 .
$$

For finite $g_{2}$, these operators mix [14-16], and the stateoperator correspondence has been worked out to order $g_{2}$ in $[6,15]$ (see also [19]). The operators which correspond to the desired one- and two-string states are

$$
\begin{aligned}
\widetilde{O}_{p}= & O_{p}-\frac{g_{2}}{2} \sum_{k=-\infty}^{\infty} \int_{0}^{1} d y C_{p k y} T_{k}^{y}-\frac{g_{2}}{2} \int_{0}^{1} d y C_{p y} T^{y} \\
& +\mathcal{O}\left(g_{2}^{2}\right), \\
\widetilde{T}_{k}^{y}= & T_{k}^{y}-\frac{g_{2}}{2} \sum_{p=-\infty}^{\infty} C_{p k y} O_{p}+g_{2}(\text { triple trace })+\mathcal{O}\left(g_{2}^{2}\right),
\end{aligned}
$$

where

$$
C_{p k y}=\sqrt{\frac{1-y}{J y}} \frac{\sin ^{2}(\pi p y)}{\pi^{2}(p-k / y)^{2}}, \quad C_{p y}=-\frac{\sin ^{2}(\pi p y)}{\sqrt{J} \pi^{2} p^{2}} .
$$

The triple-trace operators in Eq. (96) will not be important for this calculation. The anomalous dimension matrix element between the single-string state $\widetilde{O}$ and the two-string state $\widetilde{T}$ is read off from the two-point function

$$
(2 \pi x)^{\Delta_{n}+\Delta_{m}^{y}}\left\langle\overline{\widetilde{O}}_{n}(x) \widetilde{T}_{m}^{y}(0)\right\rangle=-g_{2} h_{m n y} \ln (x \Lambda)^{2} .
$$

The prediction from Eq. (93), when expressed in gauge theory variables, is simply

$$
\begin{aligned}
h_{m n y}= & \frac{H_{m n y}}{\sqrt{J y(1-y)}} \\
\approx & \frac{\lambda^{\prime}}{2} \sqrt{\frac{1-y}{J y}} \frac{\sin ^{2}(\pi n y)}{\pi^{2}}\left[1+\lambda^{\prime} n^{2}\right]^{-1 / 2} \\
& \times\left[1+\lambda^{\prime} m^{2} / y^{2}\right]^{-1 / 2},
\end{aligned}
$$

up to the overall function $v(\mu, y)$ discussed in the previous subsection. The leading $\lambda^{\prime}$ term in this result agrees with the one-loop field theory calculations of $[13,14,16]$ when the appropriate operator redefinition in Eq. (96) is taken into account. Note that since Eq. (99) is already proportional to $\lambda^{\prime}$, probing the subleading terms in this expression would require a two-loop gauge theory calculation, which has not yet been reported in the literature.

\section{CONCLUSION AND DISCUSSION}

The primary results of this paper are twofold. First, we have shown through a quite intricate analysis that it is possible to determine all Neumann coefficients in the plane wave background exactly in terms of a single function $F(\mu, y)$, for any value of the mass parameter $\mu$, and we have provided an explicit formula for $F$ in Eq. (50). Sec- ondly, we have investigated the large $\mu$ behavior of these coefficients and presented simple formulas in Sec. V F which give the Neumann matrices to all orders in a $1 / \mu$ expansion. Note that although we have not discussed the matrix elements of the prefactor (nor the fermionic Neumann matrices) in detail, they are very easily obtained from the Neumann vectors (71) using the results of $[1,2,5,7]$.

Perhaps the most remarkable fact about our results is that although the Neumann matrices are very complicated functions of $\mu$, the final expressions (84) and (85) for the $1 / \mu$ expansion are very simple. Ours was a long (and perhaps circuitous) road, and although we have derived a number of nontrivial identities along the way, one cannot help but wonder whether there is a more direct path which yields the same final result. In particular, our prediction (99) for a particular gauge theory calculation is so simple that it cries out for explanation by some clever argument, perhaps along the lines of [17].

The origin of this simplicity is the fact that the function $F(\mu, y)$ behaves for large $\mu$ like

$$
\begin{aligned}
F(\mu, y)= & -\ln [4 \pi \mu y(1-y)] \\
& +\mathcal{O}\left(e^{-2 \pi \mu}, e^{-2 \pi \mu y}, e^{-2 \pi \mu(1-y)}\right),
\end{aligned}
$$

with no perturbative corrections (i.e., inverse powers of $\mu$ ). This remarkable fact, which we have proven in two different ways (in Appendices $\mathrm{C}$ and $\mathrm{D}$ ), is reminiscent of various non-renormalization theorems. Indeed, although the BMN operators in Eq. (94) are non Bogomol'nyl-PrasadSommerfield (BPS) in general, their anomalous dimensions

$$
\Delta\left(O_{n}\right)-J-2=2 \sqrt{1+\lambda^{\prime} n^{2}}-2, \quad \lambda^{\prime}=\frac{g_{\mathrm{YM}}^{2} N}{J^{2}}
$$

are nevertheless finite in the limit of large 't Hooft coupling, provided that $J$ is simultaneously taken to infinity so that $\lambda^{\prime}$ remains finite. This suggests that there should be some residual "effective supersymmetry" protecting these operators and their interactions.

We have not worked out explicit formulas for the nonperturbative terms in Eq. (99), although these could in principle be obtained by extending the analysis of the appendices. Finally, the presence of fractional powers of $\lambda^{\prime}$ in certain string field theory observables has been noted in $[2,3]$. These surprising powers appear in matrix elements when the total number of "impurities" is not conserved. For example, a matrix element in which two impurities are created or destroyed is in general a factor of $\mu=1 / \sqrt{\lambda^{\prime}}$ larger than a similar matrix element with conserved impurity number. Although this seems enigmatic from the dual gauge theory point of view, it has been stressed $[3,18]$ that there is no reason why the large $J$ limit of the $\lambda$ expansion has to agree, order by order in $\lambda$, with the $\lambda^{\prime}$ expansionespecially in light of the fact that the BMN limit requires taking $\lambda \rightarrow \infty$, which need not be a trivial extrapolation. For the anomalous dimensions Eq. (101) it seems to work, but in order to reproduce impurity non-conserving interactions on 
the gauge theory side one might have to sum the $\lambda$ expansion to all orders and then take the large $J$ limit.

\section{ACKNOWLEDGMENTS}

M.S. and A.V. are grateful to I. Klebanov for collaboration in the early stages of their work on this project, and to D. Freedman and R. Roiban for helpful discussions. The hospitality of Princeton University (A.V.) and the Aspen Center for Physics (J.H.S., M.S. and A.V.), where portions of this work were carried out, is also appreciated. This research was supported in part by the U.S. Dept. of Energy under Grants No. DE-FG02-95ER40893 (Y.H.H.), DE-FG03-92-ER40701 (J.H.S.) and DE-FG02-91ER40671 (M.S.), and by the National Science Foundation under Grant No. PHY99-07949 (A.V.).

\section{APPENDIX A: BASIC IDENTITIES}

The infinite matrices $A_{m n}^{(r)}, C$ and the infinite vector $B_{m}$ satisfy a number of useful relations which we record here:

$$
\begin{gathered}
A^{(r) \mathrm{T}} C A^{(s)}=\frac{1}{\alpha_{r}} C \delta^{r s}, \quad r, s \in\{1,2\}, \\
A^{(r) \mathrm{T}} C^{-1} A^{(s)}=\alpha_{r} C^{-1} \delta^{r s}, \quad r, s \in\{1,2\}, \\
A^{(r) \mathrm{T}} C B=0, \quad r \in\{1,2\},
\end{gathered}
$$

and

$$
B^{\mathrm{T}} C B=\frac{2}{y(1-y)},
$$

where we have used $\alpha_{3}=-1$. Some additional useful identities are

$$
\begin{gathered}
\sum_{r=1}^{3} \frac{1}{\alpha_{r}} A^{(r)} C A^{(r) \mathrm{T}}=0, \\
\sum_{r=1}^{3} \alpha_{r} A^{(r)} C^{-1} A^{(r) \mathrm{T}}=\frac{\alpha}{2} B B^{\mathrm{T}},
\end{gathered}
$$

where, as before,

$$
\alpha=\alpha_{1} \alpha_{2} \alpha_{3}=-y(1-y) \text {. }
$$

\section{APPENDIX B: DERIVATION OF THE DIFFERENTIAL EQUATION}

In this appendix we fill in the details of the derivation in Sec. III, making frequent use of the identities presented in Appendix A.

We start by deriving Eq. (31). Using Eq. (A1) to simplify $\Gamma_{+} C A^{(r)}$ gives the identity

$$
\Gamma_{+}^{-1} C_{3} A^{(r)}=C A^{(r)}-\frac{1}{\alpha_{r}} \Gamma_{+}^{-1} A^{(r)} C_{r}, \quad r \in\{1,2\} .
$$

It follows from this that

$$
Y^{\mathrm{T}} C U_{3}^{-1} A^{(r)}=-\frac{1}{\alpha_{r}} Y^{\mathrm{T}} A^{(r)} U_{r}^{-1} C, \quad r \in\{1,2\} .
$$

Multiplying Eq. (17) on the right by $A^{(r)}$ and using Eqs. (B1) and (B2) then gives

$$
\begin{aligned}
& \Gamma_{+}^{-1} A^{(r)} C_{r}-\alpha_{r} C_{3} \Gamma_{+}^{-1} A^{(r)} \\
& \quad=-\frac{1}{2} \frac{\alpha}{1+\mu \alpha k} C U_{3}^{-1} Y Y^{\mathrm{T}} A^{(r)} U_{r}^{-1} C, \quad r \in\{1,2\} .
\end{aligned}
$$

Comparing this to Eq. (17), we can write the following formula for all $r$ :

$$
\begin{aligned}
\Gamma_{+}^{-1} A^{(r)} C_{r}-\alpha_{r} C_{3} \Gamma_{+}^{-1} A^{(r)} & \\
& =C \delta_{r 3}-\frac{1}{2} \frac{\alpha}{1+\mu \alpha k} C U_{3}^{-1} Y Y^{\mathrm{T}} A^{(r)} C U_{r}^{-1} .
\end{aligned}
$$

Multiplying this further by $B^{\mathrm{T}} C_{3}^{-1}$ on the left and by $\alpha_{r} C^{-1} C_{r}^{-1} A^{(r) \mathrm{T}}$ on the right, then summing over $r$, gives

$$
\begin{aligned}
\frac{1}{2} \alpha k_{1} B^{\mathrm{T}}-Y^{\mathrm{T}} N= & -B^{\mathrm{T}} C_{3}^{-2}-\frac{1}{2} \frac{\alpha}{1+\mu \alpha k}\left(k-\mu k_{1}\right) \\
& \times\left(\frac{1}{2} \alpha k B^{\mathrm{T}}+\mu Y^{\mathrm{T}} N\right) .
\end{aligned}
$$

The transpose of Eq. (B5) is a linear equation for $N Y$ whose solution is Eq. (31).

Next we obtain Eq. (35). We start by using a variant of Eq. (17) to obtain an identity for $\left[C_{3}^{-2}, \Gamma_{+}^{-1}\right]$ $=\left[C_{3}^{-1},\left\{\Gamma_{+}^{-1}, C_{3}^{-1}\right\}\right]$ which yields

$$
\begin{aligned}
{\left[C_{3}^{-2}, \Gamma_{+}^{-1}\right] B=} & -\frac{1}{2} \frac{\alpha}{1+\mu \alpha k}\left[\left(k-\mu k_{1}\right)\left(C_{3}^{-1}-\mu C_{3}^{-2}\right)\right. \\
& \left.-\left(k_{1}-\mu k_{2}\right)\left(1-\mu C_{3}^{-1}\right)\right] Y
\end{aligned}
$$

and hence

$$
\begin{aligned}
\Gamma_{+}^{-1} C_{3}^{-2} B= & \frac{1}{2} \frac{\alpha}{1+\mu \alpha k}\left[\left(k_{2} \mu-k_{1}\right)+\left(k-\mu^{2} k_{2}\right) C_{3}^{-1}\right. \\
& \left.+\left(\frac{2}{\alpha}+k \mu+k_{1} \mu^{2}\right) C_{3}^{-2}\right] Y .
\end{aligned}
$$

Then we can substitute Eq. (31) into Eq. (30) and use Eq. (B7) to arrive at Eq. (35).

\section{APPENDIX C: ON AN INTEGRAL TRANSFORM}

In this appendix we show that the general solution of the Fredholm integral equation of the first kind

$$
\int_{0}^{\infty} d x \frac{f(x)}{\left(x^{2}+z^{2}\right)^{3 / 2}}=g(z)
$$


is

$$
f(x)=i \frac{x^{2}}{\pi} \int_{0}^{\pi / 2}[g(i x \cos \theta)-g(-i x \cos \theta)] \cos \theta d \theta .
$$

For the amusement of the reader we present two derivations of this result. The first is a set of manipulations that indicate how we arrived at the solution, while the second is a more rigorous proof of the theorem as stated in the text.

\section{An elementary manipulation}

As integral equations of the first kind are notoriously difficult to solve, let us attempt to circumvent the problem. The method used here does not seem to be in the canonical texts and is hoped to be of some use.

Consider the following integral identity [20]:

$$
\int_{0}^{\infty} \frac{x J_{0}(x j) d x}{\left(x^{2}+z^{2}\right)^{3 / 2}}=\frac{e^{-z j}}{z}, \quad j>0, \operatorname{Re}(z)>0 .
$$

Next suppose that $z g(z)$ can be expanded as a power series in $e^{-z}-1$ :

$$
z g(z)=\sum_{j=0}^{\infty} a_{j}\left(e^{-z}-1\right)^{j}=\sum_{j=0}^{\infty} a_{j} \sum_{k=0}^{j}\left(\begin{array}{l}
j \\
k
\end{array}\right)(-1)^{j-k} e^{-z k} .
$$

Therefore by Eq. (C3), we have

$$
\begin{aligned}
g(z) & =\sum_{j=0}^{\infty} a_{j} \sum_{k=0}^{j}\left(\begin{array}{l}
J \\
k
\end{array}\right)(-1)^{j-k} \frac{e^{-z k}}{z} \\
& =\sum_{j=0}^{\infty} a_{j} \sum_{k=0}^{j}\left(\begin{array}{l}
J \\
k
\end{array}\right)(-1)^{j-k} \int_{0}^{\infty} \frac{x J_{0}(x k) d x}{\left(x^{2}+z^{2}\right)^{3 / 2}},
\end{aligned}
$$

from which we can directly read off the solution

$$
f(x)=x \sum_{j=0}^{\infty} a_{j} \sum_{k=0}^{j}\left(\begin{array}{l}
j \\
k
\end{array}\right)(-1)^{j-k} J_{0}(x k) .
$$

This formidable sum can actually be performed due to the identity [20]

$$
J_{0}(k x)=\frac{1}{\pi} \int_{0}^{\pi} e^{i k x \cos \theta} d \theta
$$

from which we obtain

$$
\begin{aligned}
f(x) & =\frac{x}{\pi} \sum_{j=0}^{\infty} a_{j} \sum_{k=0}^{j}\left(\begin{array}{l}
j \\
k
\end{array}\right)(-1)^{j-k} \int_{0}^{\pi}\left(e^{i x \cos \theta}\right)^{k} d \theta \\
& =\frac{x}{\pi} \int_{0}^{\pi} \sum_{j=0}^{\infty} a_{j}\left(e^{i \cos x \theta}-1\right)^{j} d \theta \\
& =\frac{-i x^{2}}{\pi} \int_{0}^{\pi} d \theta \cos \theta g(-i x \cos \theta) .
\end{aligned}
$$

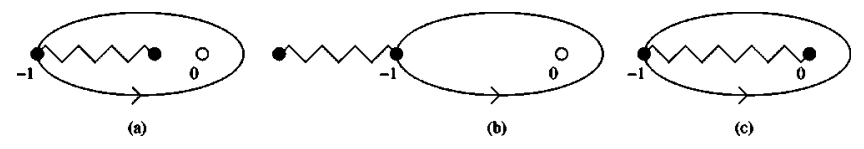

FIG. 1. The contour $\mathcal{C}$ for Appendices C and D. In (a) and (b) we show the singularity structure of Eq. (C8), with branch points at -1 and $-y^{\prime} / y$. In (c) we show the structure of Eqs. (C11) (for integer $k)$ and (D4).

Therefore all dependence on the coefficients $a_{j}$ drops out and we have a generalized Fourier transform of rather simple form.

The main shortcoming of this derivation is that it assumes the existence of a rather peculiar expansion, which might not be necessary. The alternative approach presented in the remainder of this appendix is clearer in this regard.

\section{Representation of a delta function}

Here we consider the integral

$$
\chi\left(y, y^{\prime}\right)=\frac{\sqrt{y}}{4 \pi i} \int_{\mathcal{C}} \frac{d w}{\sqrt{1+w}} \frac{1}{\left(w y+y^{\prime}\right)^{3 / 2}},
$$

where $y, y^{\prime}>0$ and $\mathcal{C}$ is the contour shown in Fig. 1. For $y^{\prime}<y$ the singularity structure is shown in Fig. 1(a), and the contour can be pushed off to infinity, giving zero for the integral. For $y^{\prime}>y$, the contour encloses no singularity [Fig. $1(\mathrm{~b})]$, so the result is again zero.

Since $\chi\left(y, y^{\prime}\right)$ vanishes for $y^{\prime} \neq y$, let us check whether it is a delta function (and in particular, that it does not involve any derivatives of delta functions) by integrating it against the test function $e^{-t y^{\prime}}$ :

$$
\begin{aligned}
\int_{0}^{\infty} d y^{\prime} \chi\left(y, y^{\prime}\right) e^{-t y^{\prime}}= & \frac{1}{2 \pi i} \int_{\mathcal{C}} \frac{d w}{\sqrt{1+w} \sqrt{w}}\left[1+e^{t w y}\right. \\
& \times \sqrt{\pi t w y}(\Phi(\sqrt{t w y})-1)]
\end{aligned}
$$

where the result of the $y^{\prime}$ integral involves the error function

$$
\Phi(x)=\frac{2}{\sqrt{\pi}} \int_{0}^{x} e^{-t^{2}} d t .
$$

Next we make use of the elementary integral

$$
\frac{1}{2 \pi i} \int_{\mathcal{C}} \frac{d w}{\sqrt{1+w} \sqrt{w}} w^{k}=\left(\begin{array}{c}
-\frac{1}{2} \\
k
\end{array}\right) .
$$

In particular, note that this is zero when $k$ is a positive halfinteger since the branch cut then runs from -1 to $-\infty$, so there is no singularity within the contour. The term in Eq. (C9) proportional to " -1 " therefore integrates to zero, since it contains only half-integer powers of $w$. For the remaining terms we use the expansion 


$$
1+e^{x} \sqrt{\pi x} \Phi(\sqrt{x})=\sum_{k=0}^{\infty} \frac{2^{k} x^{k}}{(2 k-1) ! !}
$$

Note that $(-1) ! !=1$. Combining Eqs. (C12) and (C11) leads to

$$
\begin{aligned}
\int_{0}^{\infty} d y^{\prime} \chi\left(y, y^{\prime}\right) e^{-t y^{\prime}} & =\sum_{k=0}^{\infty} \frac{2^{k}(t y)^{k}}{(2 k-1) ! !}\left(\begin{array}{c}
-\frac{1}{2} \\
k
\end{array}\right) \\
& =\sum_{k=0}^{\infty} \frac{(-1)^{k}}{k !}(t y)^{k}=e^{-t y}
\end{aligned}
$$

where we have used the identity $(2 k-1) ! !=$ $(-2)^{k} k !\left(\begin{array}{c}-1 / 2 \\ k\end{array}\right)$. If $\chi$ contained any terms proportional to derivatives of delta-functions, we would have obtained a polynomial in $t$ times $e^{-t y}$ in Eq. (C13). Since this did not happen, we have proven that

$$
\begin{aligned}
\chi\left(y, y^{\prime}\right) & =\frac{\sqrt{y}}{4 \pi i} \int_{\mathcal{C}} \frac{d w}{\sqrt{1+w}} \frac{1}{\left(w y+y^{\prime}\right)^{3 / 2}} \\
& =\delta\left(y-y^{\prime}\right), \quad y, y^{\prime}>0 .
\end{aligned}
$$

More precisely, we have proved that $\int \chi\left(y, y^{\prime}\right) f\left(y^{\prime}\right) d y^{\prime}$ $=f(y)$ for any $f$ that can be written as a convergent Laplace transform. It is not excluded that an even weaker condition would suffice.

\section{Formal proof}

To verify our solution let us substitute $f(x)$ from Eq. (C2) into the integral equation $(\mathrm{C} 1)$ and replace the integration variable $x$ by $t=x \cos \theta$. This gives

$$
\begin{gathered}
-\frac{i}{\pi} \int_{0}^{\infty} d t \int_{0}^{\pi / 2} d \theta \frac{t^{2}[g(-i t)-g(i t)] \cos \theta}{\left(t^{2}+z^{2} \cos ^{2} \theta\right)^{3 / 2}} \\
\quad=-\frac{i}{\pi} \int_{0}^{\infty} d t \frac{t[g(-i t)-g(i t)]}{\left(t^{2}+z^{2}\right)}
\end{gathered}
$$

where we have used

$$
\int_{0}^{\pi / 2} \frac{\cos \theta d \theta}{\left(t^{2}+z^{2} \cos ^{2} \theta\right)^{3 / 2}}=\frac{1}{t\left(t^{2}+z^{2}\right)} .
$$

We can now recast the remaining integral in Eq. (C15) as a contour integral with the contour $\mathcal{C}^{\prime}$ enclosing the positive $u$ axis. This gives

$$
\frac{1}{2 \pi i} \int_{\mathcal{C}^{\prime}} d u \frac{g(-i \sqrt{u})}{\left(u+z^{2}\right)}=g(z),
$$

where we have used the assumed analytic and asymptotic properties of $g$, which are just what are needed to deform the contour into one that encircles the pole at $-z^{2}$, thereby reducing the integral to exactly $g(z)$.

We have proven that the function $f(x)$ given in Eq. (C2), when substituted into Eq. (C1), indeed gives $g(z)$. It remains to show that the result is unique, in other words that when $g(z)$ from Eq. (C1) is substituted into Eq. (C2), we recover $f(x)$. To this end, we define first

$$
f(x)=x \widetilde{f}\left(x^{2}\right)
$$

and let $v^{\prime}=x^{2}$ so that the integral equation $(\mathrm{C} 1)$ becomes

$$
\frac{1}{2} \int_{0}^{\infty} d v^{\prime}\left(z^{2}+v^{\prime}\right)^{-3 / 2} \widetilde{f}\left(v^{\prime}\right)=g(z) .
$$

Our proposed solution is, in terms of $\widetilde{f}(v)$,

$$
\widetilde{f}(v)=-\frac{i \sqrt{v}}{\pi} \int_{0}^{\pi} g(-i \sqrt{v} \cos \theta) \cos \theta d \theta .
$$

Letting $\sqrt{w}=-i \cos \theta$ gives

$$
\widetilde{f}(v)=\frac{\sqrt{v}}{2 \pi i} \int_{\mathcal{C}} \frac{d w}{\sqrt{1+w}} g(\sqrt{w v}),
$$

where the contour $\mathcal{C}$ is as in Fig. 1. Substituting Eq. (C1) for $g(z)$ and using the delta-function representation Eq. (C14) establishes Eq. (C21) and hence completes the proof.

\section{APPENDIX D: ASYMPTOTIC BEHAVIOR OF $\boldsymbol{F}(\boldsymbol{\mu}, \boldsymbol{y})$}

The analysis of Appendix C was completely general, and we only used rather weak assumptions about the form of $g(z)$. Here we use the contour integral techniques above to study the particular case of interest, with $g(z)$ given by $G(z, y)$ from Eq. (40). Recall Stirling's asymptotic expansion

$$
\ln \Gamma(1+z) \approx\left(z+\frac{1}{2}\right) \ln z-z+\frac{1}{2} \ln 2 \pi+\sum_{n=0}^{\infty} \frac{c_{n}}{z^{2 n+1}} .
$$

The coefficients $c_{n}$ may be expressed in terms of Bernoulli numbers, but they will turn out to be irrelevant. The crucial fact is that only odd inverse powers of $z$ appear in Eq. (D1). It follows that

$$
G(z, y) \approx-\frac{1}{z^{2}} \ln (2 \pi z y(1-y))+\sum_{n=1}^{\infty} \frac{b_{n}(y)}{z^{2 n+1}} .
$$

Now letting $\sqrt{w}=-i \cos \theta$ in Eq. (43), as in the previous subsection, gives

$$
F(\mu, y)=\frac{\mu^{2}}{2 \pi i} \int_{\mathcal{C}} \frac{d w}{\sqrt{1+w}} G(\mu \sqrt{w}, y) .
$$

Consider first the inverse odd powers in Eq. (D2). They lead to integrals of the form 


$$
\int_{\mathcal{C}} \frac{d w}{\sqrt{1+w}} \frac{1}{w^{n+1 / 2}}
$$

for $n$ a positive integer. The branch cut is now as shown in Fig. 1(c), so the contour may be pulled off to infinity, giving zero. Therefore only the first term in Eq. (D2) contributes, so

$$
\begin{aligned}
F(\mu, y) \approx & -\frac{1}{2 \pi i} \int_{\mathcal{C}} \frac{d w}{w \sqrt{1+w}} \ln (2 \pi \mu \sqrt{w} y(1-y)) \\
\approx & -\ln (2 \pi \mu y(1-y)) \frac{1}{2 \pi i} \int_{\mathcal{C}} \frac{d w}{w \sqrt{1+w}} \\
& -\frac{1}{4 \pi i} \int_{\mathcal{C}} \frac{\ln w}{w \sqrt{1+w}} d w .
\end{aligned}
$$

The first integral gives 1 since it just picks off the pole at $w=0$. It is an interesting though straightforward exercise to check that the second term gives $-\ln 2$. This completes the direct proof of Eq. (60).

\section{APPENDIX E: ADVANCED SUMS}

Let us review an elementary trick which can be used to evaluate certain infinite sums. Consider first the sum

$$
\sum_{n=-\infty}^{\infty} f(n), \quad f(z)=\frac{1}{z^{2}+x^{2} v^{2}} \frac{1}{\sqrt{z^{2}+x^{2}}}
$$

for $x>0$ and $v>1$. This sum can be written as the contour integral

$$
\frac{1}{2 \pi i} \int_{\mathcal{C}} d z f(z) \pi \cot \pi z
$$

where $\mathcal{C}$ passes from $-\infty-i \epsilon$ to $+\infty-i \epsilon$ slightly below the real axis, and then returns slightly above the real axis (see Fig. 2). The contour may be deformed away from the poles of $f(z) \pi \cot \pi z$ on the real axis to pick up the other singularities instead. In this case the only other singularities are branch cuts from $\pm i x$ to $\pm i \infty$, and poles at $z= \pm i x v$ sitting on the branch cuts. Rescaling $z$ and combining the two cuts, we find therefore that

$$
\sum_{n=-\infty}^{\infty} \frac{1}{n^{2}+x^{2} v^{2}} \frac{1}{\sqrt{n^{2}+x^{2}}}=-\frac{2}{x^{2}} \mathrm{P} \int_{1}^{\infty} \frac{d z}{\sqrt{z^{2}-1}} \frac{\operatorname{coth}(\pi x z)}{z^{2}-v^{2}}
$$

where $\mathrm{P}$ stands for the principal value. This is an exact formula, but if we are not concerned with terms vanishing exponentially for large $x$, then we can $\operatorname{set} \operatorname{coth}(\pi x z)=1$ in the integral, which is then easily evaluated to give

$$
\sum_{n=-\infty}^{\infty} \frac{1}{n^{2}+x^{2} v^{2}} \frac{1}{\sqrt{n^{2}+x^{2}}} \approx \frac{2}{x^{2}} \frac{\operatorname{arccosh}(v)}{v \sqrt{v^{2}-1}}
$$

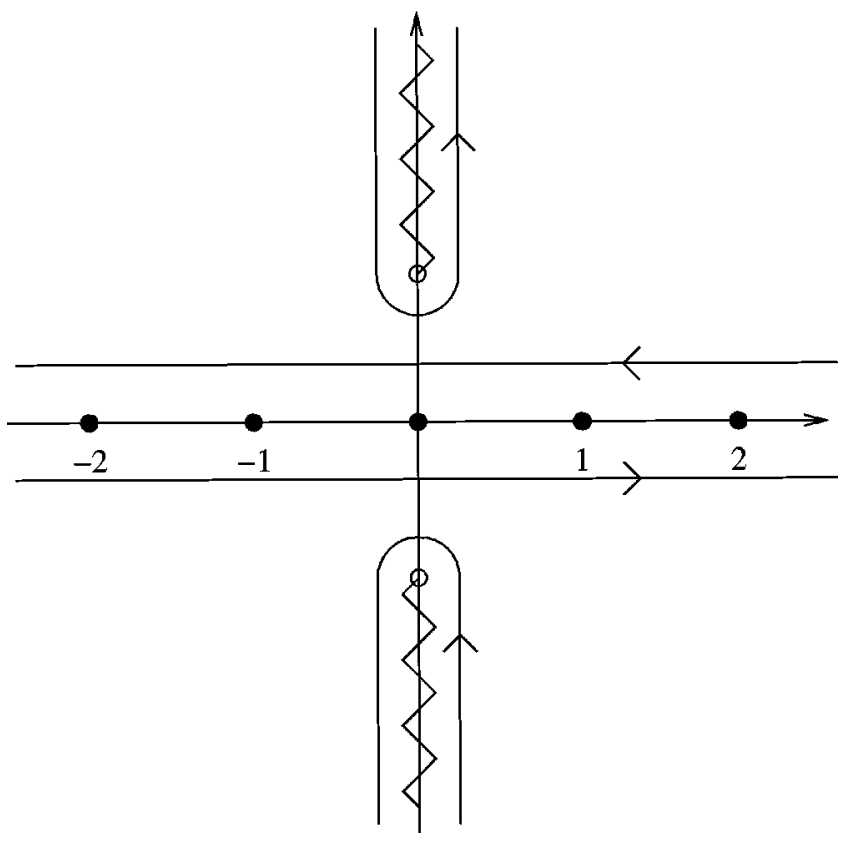

FIG. 2. This figure shows the contours relevant for analyzing the various sums and integrals in Appendix E. In each case, the contour is deformed from the one enclosing the real axis to the one enclosing the branch cuts on the imaginary axis. Note that the integrands in Eqs. (E3) and (E11) have two additional poles on top of the branch cuts, while the integrand in Eq. (E7) has two additional poles on the real axis.

As in Sec. $\mathrm{V}$, the symbol $\approx$ denotes that we have dropped terms of order $e^{-2 \pi x}$. Taking the limit $v \rightarrow 1$ from above finally gives the result

$$
\sum_{n=-\infty}^{\infty} \frac{1}{\left(n^{2}+x^{2}\right)^{3 / 2}} \approx \frac{2}{x^{2}} .
$$

For sums in which $f(z)$ itself contains trigonometric functions, it is convenient to expand these and write the sum in the form

$$
\sum_{n=-\infty}^{\infty}\left[f_{1}(n)+(-1)^{n} f_{2}(n)\right]
$$

The term with $f_{1}(z)$ can be evaluated using the above trick, while the second term can be evaluated by deforming the contour integral of $f_{2}(z) \pi \csc \pi z$. Applying this method and taking into account both the contribution from the poles at $\pm n / y$ and the discontinuity across the branch cuts gives

$$
\begin{aligned}
\sum_{m=1}^{\infty} & \frac{\sin ^{2}(\pi m y)}{m^{2}-n^{2} / y^{2}} \frac{\sqrt{x+\sqrt{x^{2}+m^{2}}}}{\sqrt{x^{2}+m^{2}}} \\
& =-\frac{1}{2 x^{3 / 2}} \operatorname{Re} \int_{1}^{\infty} \frac{d z}{\sqrt{z^{2}-1}} \frac{\sqrt{1+i \sqrt{z^{2}-1}}}{z^{2}+n^{2} /\left(x^{2} y^{2}\right)} \frac{1}{F(x, y, z)}
\end{aligned}
$$

when $0<y<1, x>0$, and $n$ is a non-zero integer. We have defined the function 


$$
F(x, y, z)=\frac{1}{2}[\operatorname{coth}(\pi x y z)+\operatorname{coth}(\pi x(1-y) z)] .
$$

Ignoring exponentially small corrections, we can set $F=1$ and evaluate the integral in Eq. (E7) explicitly, arriving at

$$
\begin{aligned}
& \sum_{m=1}^{\infty} \frac{\sin ^{2}(\pi m y)}{m^{2}-n^{2} / y^{2}} \frac{\sqrt{x+\sqrt{x^{2}+m^{2}}}}{\sqrt{x^{2}+m^{2}}} \\
& \quad \approx-\frac{\pi y^{3 / 2}}{4 n} \frac{\sqrt{\sqrt{n^{2}+x^{2} y^{2}}-x y}}{\sqrt{n^{2}+x^{2} y^{2}}} .
\end{aligned}
$$

The case $n=0$ must be considered separately since the poles at $m^{2}= \pm n / y$ are then lost. The result in this case is

$$
\sum_{m=1}^{\infty} \frac{\sin ^{2}(\pi m y)}{m^{2}} \frac{\sqrt{x+\sqrt{x^{2}+m^{2}}}}{\sqrt{x^{2}+m^{2}}} \approx \frac{\pi^{2} y(1-y)}{\sqrt{2 x}}-\frac{\pi}{4 \sqrt{2} x^{3 / 2}} .
$$

A combination of all of the above techniques is needed to tackle the final sum

$$
\begin{aligned}
& \sum_{m=1}^{\infty} \frac{\sin ^{2}(\pi m y)}{m^{2}+x^{2} v^{2}} \frac{\sqrt{x+\sqrt{x^{2}+m^{2}}}}{\sqrt{x^{2}+m^{2}}} \\
& \approx-\frac{2}{x^{3 / 2}} \operatorname{ReP} \int_{1}^{\infty} \frac{d z}{\sqrt{z^{2}-1}} \frac{\sqrt{1+i \sqrt{z^{2}-1}}}{z^{2}-v^{2}} \\
& =-\frac{\pi}{2 x^{3 / 2}} \frac{\operatorname{Im} \sqrt{1-i \sqrt{v^{2}-1}}}{v \sqrt{v^{2}-1}},
\end{aligned}
$$

which is valid for $v>1$

\section{APPENDIX F: SOME EXPONENTIAL CORRECTIONS}

In this appendix we derive the exact formula (61) which allows systematic determination of the exponential corrections to $F(\mu, y)$. It follows from Eq. (E3) that

$$
\phi^{\prime \prime}(x)=-x \sum_{n=1}^{\infty} \frac{1}{\left(x^{2}+n^{2}\right)^{3 / 2}}=\frac{1}{2 x^{2}}+\frac{1}{x} I(x)
$$

where

$$
\begin{aligned}
I(x)= & \mathrm{P} \int_{1}^{\infty} \frac{\operatorname{coth}(\pi x z)}{\left(z^{2}-1\right)^{3 / 2}} d z=-\frac{1}{2} \oint_{|z-1|=\epsilon} \frac{\operatorname{coth}(\pi x z)}{\left(z^{2}-1\right)^{3 / 2}} d z \\
& +\int_{1+\epsilon}^{\infty} \frac{\operatorname{coth}(\pi x z)}{\left(z^{2}-1\right)^{3 / 2}} d z .
\end{aligned}
$$

Integrating the second term by parts gives a divergent piece that cancels the divergent piece from the first term, leaving

$$
I(x)=-1-\pi x \int_{1}^{\infty} \frac{z d z}{\sqrt{z^{2}-1}} \frac{1}{\sinh ^{2}(\pi x z)} .
$$

Therefore

$$
\phi^{\prime \prime}(x)=-\frac{1}{x}+\frac{1}{2 x^{2}}-\pi \int_{1}^{\infty} \frac{z d z}{\sqrt{z^{2}-1}} \frac{1}{\sinh ^{2}(\pi x z)} .
$$

Integrating twice with respect to $x$ as in Sec. V A and using Eq. (50) yields the formulas (61) and (62).
[1] M. Spradlin and A. Volovich, Phys. Rev. D 66, 086004 (2002).

[2] M. Spradlin and A. Volovich, J. High Energy Phys. 01, 036 (2003).

[3] I.R. Klebanov, M. Spradlin, and A. Volovich, Phys. Lett. B 548, 111 (2002).

[4] J.H. Schwarz, J. High Energy Phys. 09, 058 (2002).

[5] A. Pankiewicz, J. High Energy Phys. 09, 056 (2002).

[6] J. Pearson, M. Spradlin, D. Vaman, H. Verlinde, and A. Volovich, "Tracing the string: BMN correspondence at finite $J^{2} / N$," hep-th/0210102.

[7] A. Pankiewicz and B. Stefański, Jr., "pp-wave light-cone superstring field theory," hep-th/0210246.

[8] D. Berenstein, J.M. Maldacena, and H. Nastase, J. High Energy Phys. 04, 013 (2002)

[9] M.B. Green and J.H. Schwarz, Nucl. Phys. B218, 43 (1983).

[10] M.B. Green, J.H. Schwarz, and L. Brink, Nucl. Phys. B219, 437 (1983)

[11] Y. Kiem, Y. Kim, S. Lee, and J. Park, Nucl. Phys. B642, 389 (2002).
[12] M. Huang, Phys. Lett. B 542, 255 (2002).

[13] C.S. Chu, V.V. Khoze, and G. Travaglini, J. High Energy Phys. 06, 011 (2002).

[14] N. Beisert, C. Kristjansen, J. Plefka, G.W. Semenoff, and M. Staudacher, Nucl. Phys. B650, 125 (2003).

[15] D.J. Gross, A. Mikhailov, and R. Roiban, "A calculation of the plane wave string Hamiltonian from $\mathrm{N}=4$ super-Yang-Mills theory," hep-th/0208231.

[16] N.R. Constable, D.Z. Freedman, M. Headrick, and S. Minwalla, J. High Energy Phys. 10, 068 (2002).

[17] A. Santambrogio and D. Zanon, Phys. Lett. B 545, 425 (2002).

[18] D. Vaman and H. Verlinde, "Bit strings from $\mathrm{N}=4$ gauge theory," hep-th/0209215.

[19] J. Gomis, S. Moriyama, and J. Park, "SYM description of SFT Hamiltonian in a pp-wave background," hep-th/0210153.

[20] I.S. Gradshteyn and I.M. Ryzhik, in Table of Integrals, Series, and Products, sixth ed., edited by Alan Jeffrey (Academic, New York, 2000). 